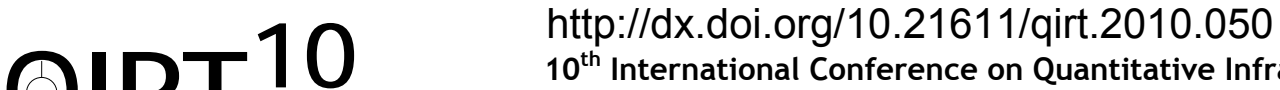 \\ $10^{\text {th }}$ International Conference on Quantitative InfraRed Thermography \\ July 27-30, 2010, Québec (Canada)
}

\section{Development of a hybrid non-destructive inspection system combining induction thermography and eddy current techniques.}

by M. Grenier*, C. Ibarra-Castanedo* and X. Maldague*

*ECE Dept., Université Laval, Québec (Quebec), Canada G1V OA6, marc.grenier.3@ulaval.ca, clemente.ibarracastanedo.1@ulaval.ca, Xavier.Maldaque@gel.ulaval.ca

\begin{abstract}
Arial, 9pt, bold)
Research conducted on induction thermography has attracted the attention of many researchers in recent years. Up to now, the electromagnetic induction phenomenon has only been used to create thermal waves in the specimen in order to carry out the inspection with an infrared camera. However, the large amount of current induced in the material can also be used to perform eddy current inspection. This paper describes a new Hybrid system that can perform Induction Thermography and Eddy Current inspection simultaneously (HITEC).
\end{abstract}

\section{Introduction (Arial, 9pt, bold)}

There are several non-destructive inspection techniques on the market that can be used to solve different applications. However, each technique has its limitations and does not always provide a complete detection of the defects present in a specimen. A way to improve the detection capability is to use multiple inspection techniques [1,2]. These techniques can be sensitive to different types of defects, or conversely, detect the same defects in order to improve the detection reliability and the sizing. Unfortunately, an inspection strategy based on multiple techniques applied successively is usually more expensive and time consuming. The idea behind the HITEC system is to take advantage of the synergy between the induction thermography and the eddy current techniques to perform two different inspections simultaneously. These two inspection techniques use the concept of electromagnetic induction to generate high density currents in the specimen. The interaction of the induced currents with the defects produces local magnetic field variations and heat than can be measured respectively with an eddy current instrument and an infrared camera. However, even if both techniques share the same excitation source, they are normally implemented in a very different way. Most induction thermography systems are used in static mode. The electromagnetic generator is excited with a burst or a modulated signal and the thermal images are acquired during the cooling down period of the material [3-5]. On the other hand, eddy current instruments are operated in dynamic mode. This means that the eddy current probe has to be moved over the specimen during the data acquisition. Several sensors can be placed in the eddy current probe to allow a larger coverage and to produce an image representing the electromagnetic response of the specimen [6]. The following section presents the first prototype of the HITEC measurement system and some preliminary results for two different applications.

\section{Experimental setup and results}

Figure 1 shows a schematic representation of the HITEC system (figure 1a) and the laboratory setup currently used for the inspection (figure 1b). The main component is the electromagnetic generator coil, winded around a horseshoe shape ferrite that concentrates the induced currents in the specimen. This generator produces a uniform line heating that allows thermal measurement with the infrared camera. The eddy current array sensor is placed inside the generator to measure the electromagnetic field variations coming from the defects. All those components are moving together over the surface.

Depending on the electromagnetic and thermal properties of the material inspected, the penetration depth of each technique may be quite different. For example, in ferromagnetic material such as carbon steel, even if the eddy currents are limited to the surface due to the skin effect, heat can diffuse much deeper to provide a good sensitivity to corrosion. The behavior of the system is totally different on composite material such has one made with an aluminum honeycomb core and a carbon fiber skin. In this case, the eddy currents are induced directly in the aluminum core [7]. Heat diffuses from the core toward the surface allowing the induction thermography technique to detect core damage, core unbound, excess of adhesive, and delamination (figure 2a). The eddy current technique is only sensitive to the defect affecting the core itself (figure $2 b$ ). This information provided by the eddy current can be used to confirm the position and the nature of the defect found by the induction thermography. Additional experimental results for ferromagnetic material will be presented on the final version of this paper and during the conference. 


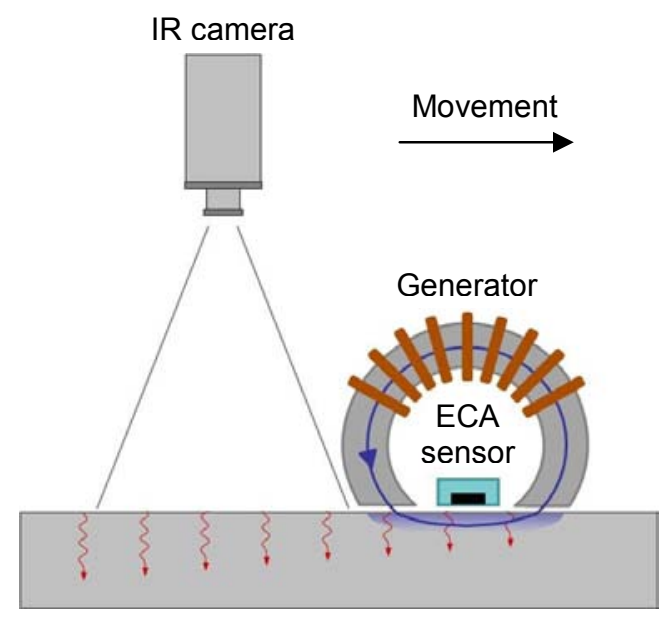

a)

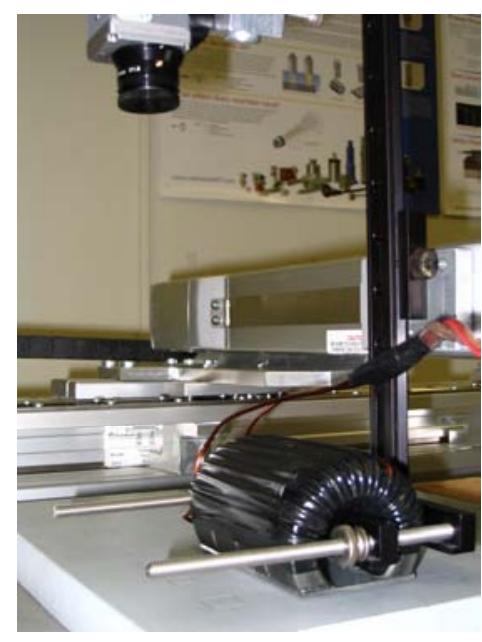

b)

Fig. 1. HITEC system configuration: a) schematic representation and b) laboratory setup.

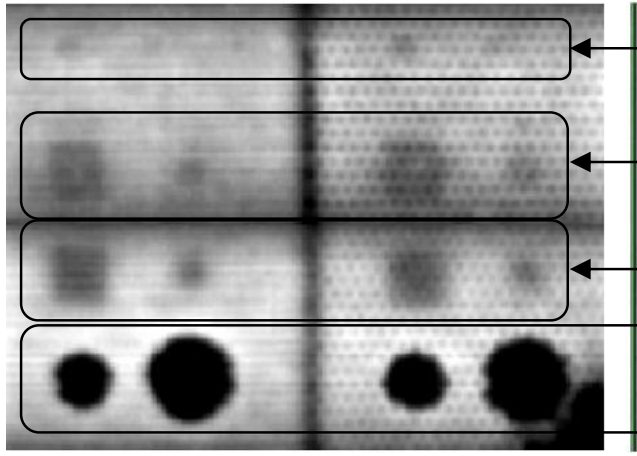

a)

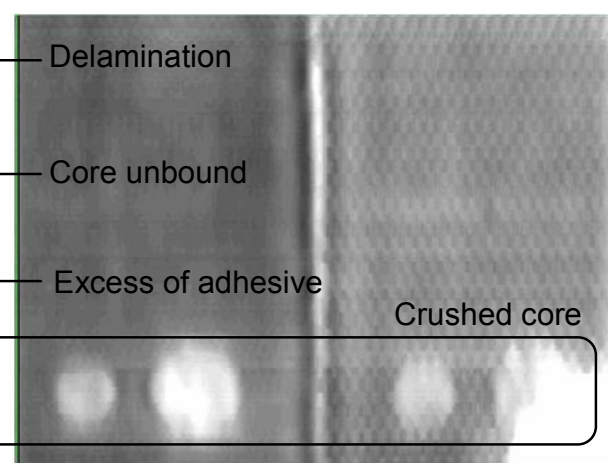

b)

Fig. 2. Composite sample made of honeycomb aluminum core and carbon fiber skin. a) results with induction thermography and b) results with eddy current array.

\section{REFERENCES}

[1] R. Edwards, A. Sophian, S. Dixon, and G. Tian, "Data fusion for combining techniques to detect and size surface and near-surface defects," Review of Progress in Quantitative Nondestructive Evaluation, 30 July-4 Aug. 2006, Portland, OR, USA: AIP, 2007, pp. 619-26.

[2] Z. Liu, Z. Liu, D.S. Forsyth, J.P. Komorowski, K. Hanasaki, and T. Kirubarajan, "Survey: State of the Art in NDE Data Fusion Techniques," Instrumentation and Measurement, IEEE Transactions on, vol. 56, 2007, pp. 2435-2451.

[3] G. Zenzinger, J. Bamberg, W. Satzger, and V. Carl, "Thermographic crack detection by eddy current excitation," Nondestructive Testing and Evaluation, vol. 22, 2007, pp. 101-111.

[4] G. Riegert, A. Gleiter, and G. Busse, "Potential and limitations of eddy current lockin-thermography," Thermosense XXVIII, Apr 17-20 2006, Kissimmee, FL, United States: International Society for Optical Engineering, Bellingham WA, WA 98227-0010, United States, 2006, p. 62051.

[5] B. Oswald-Tranta, "Thermo-inductive crack detection," Nondestructive Testing and Evaluation, vol. 22, 2007, pp. 137153.

[6] A. Chahbaz, M. Grenier, E. Peltier, and T. Bourgelas, "Fatigue crack detection in aircraft skin structures using eddy current array technique," CINDE Journal, vol. 25, Nov. 2004, pp. 14-17.

[7] R.W. McCullough, "Transient thermographic technique for NDI of aerospace composite structures," Thermosense XXVI, Apr 13-15 2004, Orlando, FL, United States: The International Society for Optical Engineering, Bellingham, United States, 2004, pp. 390-402. 